Sinergias educativas

ISSN: 2661-6661

compasacademico@icloud.com

Grupo Compás

Ecuador

\title{
Entrevista: Chiapas, espacio de retos para la investigación educativa: Luis Alan Acuña Gamboa
}

\author{
Fabián \\ Entrevista: Chiapas, espacio de retos para la investigación educativa: Luis Alan Acuña Gamboa \\ Sinergias educativas, vol. 2, núm. 2, 2017 \\ Grupo Compás, Ecuador \\ Disponible en: http://www.redalyc.org/articulo.oa?id=573561679007
}

Esta obra está bajo una Licencia Creative Commons Atribución-NoComercial-SinDerivar 4.0 Internacional. 


\section{Entrevista: Chiapas, espacio de retos para la investigación educativa: Luis Alan Acuña Gamboa}

Fabián

Ecuador

http://orcid.org/0000-0002-6264-2493

Sinergias educativas, vol. 2, núm. 2, 2017

Grupo Compás, Ecuador

Recepción: 21 Enero 2018

Aprobación: 11 Mayo 2018

Redalyc: http://www.redalyc.org/ articulo.oa?id $=573561679007$
CC BY-NC-ND
Una experiencia muy particular fue la que se vivio\# en marzo pasado, en la Universidad Auto\#noma de Chiapas. Luis Bonilla-Molina y Herman Van den Velde visitaron el estado para ofrecer los Cursos Internacionales para Investigadores en Educacio\#n, los cuales fueron avalados por el Instituto Internacional de la Unesco para la Educacio\#n Superior en Ame\# rica Latina y el Caribe (IESALC-UNESCO) y el Grupo de Trabajo del Consejo Latinoamericano de Ciencias Sociales (GT-CLACSO). Ambos investigadores de renombre, cada uno con posturas cri\#ticas respecto a las poli\#ticas implementadas en el a\#mbito educativo (por ejemplo, la teori\#a del apago\#n pedago\#gico, impulsada por BonillaMolina), ofrecieron estos cursos "rela\#mpago" que significaron una interesante ventana para conocer que\# se esta\# cociendo en los ci\# rculos acade\#micos de avanzada en el a\#mbito educativo, las tendencias de la educacio\#n a nivel global y, por supuesto, las posturas poli\#ticas de ambas personalidades, quienes reconocen la necesidad del alto compromiso social de este materia. Asi\# pues, entrevistamos a Luis Alan Acun\#a Gamboa, doctor en Estudios Regionales por la UNACH y fundador de la revista electro\#nica Sinergias educativas, responsable del evento, quien tiene todo el intere\#s de que la investigacio\#n en materia educativa encuentre un impulso, ma\#s alla\# de meritocracias ramplonas.

Fabia\#n Rivera Jua\#rez. En primer lugar, ¿co\#mo fue el proceso de gestio\#n y organizacio\#n de los cursos y cua\#les eran las expectativas del equipo de Sinergias Educativas?

Luis Alan Acun\#a Gamboa. Sin temor de equivocacio\#n, Chiapas ha necesitado a largo de su historia y, lamentablemente, au\#n en su presente, de actividades que aperturen espacios para la formacio\#n de investigadores en el campo educativo; a su vez, de contar con las posibilidades de continuar capacitando y desarrollando habilidades en la parca comunidad investigativa en esta a\#rea de la produccio\#n de conocimiento cienti\#fico. Por ello, como director general de la revista electro\#nica Sinergias Educativas y de varios colegas de e\#sta, nos surgio\# el intere\#s de ofertar, por primera vez en el estado, la imparticio\#n de cursos de calidad y reconocimiento internacional que contribuyeran a los objetivos antes mencionados. 
Asi\#, nos dimos a la tarea de gestionar, organizar y concretar, en colaboracio\#n con el Mtro. Oswaldo Antonio Gonza\#lez, la participacio\#n de los Dres. Luis Bonilla-Molina, Presidente del IESALC-UNESCO y Coordinador Internacional del Grupo de Trabajo CLACSO Reformas y contrarreformas educativas en Nuestra Ame\# rica; y Herman Van de Velde, Coordinador

de ABACO en RED y miembro del Grupo de Trabajo CLACSO Reformas y contrarreformas educativas en Nuestra Ame\#rica, que se realizo\# el pasado 10 de marzo de 2017 en las instalaciones de la Biblioteca Central de la Universidad Auto\#noma de Chiapas. Aunque el evento fue todo un e\#xito, puesto que se superaron todas las expectativas que se teni\#an en torno a los Cursos Internacionales, su realizacio\#n significo\# un trabajo colegiado de ma\#s de siete meses de preparacio\#n logi\#stica, organizativa e intelectual; esto por las agendas de los participantes, asi\# como la disponibilidad de ellos de venir a Chiapas, la cual siempre fue de alta estima e intere\#s. El cupo de los dos grupos estuvo al ma\#ximo (ya que eran limitados a 35 personas por curso), y nos obliga a pensar en la realizacio\#n de una segunda jornada, esto por la demanda que tuvieron y por la gran cantidad de interesados que no pudieron inscribirse por falta de espacios; por ello, la revista electro\#nica Sinergias Educativas se compromete en realizar nuevamente el trabajo necesario para esta segunda jornada.

F. R. J. Luis Bonilla-Molina y Herman Van den Velde, ¿cua\#l es la importancia de estas dos personalidades en el a\# mbito de la investigacio\#n enfocada al a\#mbito educativo?

L. A. A. G. Tanto Luis Bonilla-Molina como Herman Van de Velde piensan que la investigacio\#n educativa debe responder a la necesidad de buscar nuevas formas de comprender la realidad social por la que atraviesa Ame\#rica Latina y el Caribe en temas educativos y, con base en esto, proponer alternativas para solucionar los problemas educativos regionales. Para lograr esto, es imprescindible concebir que el investigador en educacio\#n, como el caso de los Dres. y quien te responde estimado Fabia\#n, debe priorizar su talante profesional bajo principios de humanismo, justicia social y derechos, priorizando los educativos, que garanticen que lo que se investiga y las conclusiones a las que se llega como producto de sus pesquisas, sirvan como insumo para mejorar los contextos socio-educativos de la regio\#n. Es por ello que la participacio\#n de Luis Bonilla-Molina y Herman Van de Velde resultan tan importantes en estados donde, como el caso chiapaneco, urge la proliferacio\#n de investigadores educativos con estas cualidades; es decir, profesionales que vean en la investigacio\#n, ma\#s alla\# del aspecto econo\#mico, la condicio\#n altruista de su talante. Sin duda alguna, los comentarios y aportes realizados durante los Cursos Internacionales IESALC- UNESCO, GT-CLACSO y ABACO en Red permitieron sembrar la simiente en cada uno de sus participantes.

F. R. J. En la escala del 0 al 10, ¿en que\# lugar se encuentra Chiapas en el a\#mbito de la investigacio\#n educativa? 
L. A. A. G. Chiapas es uno de los estados con menor I\#ndice de Desarrollo Humano, lo cual tambie\#n se evidencia en la produccio\#n de conocimiento que se genera en el campo de la educacio\#n. En una investigacio\#n reciente, expongo la situacio\#n por la que atraviesa el estado en relacio\#n a la formacio\#n de investigadores educativos, concluyendo que la realidad es poco alentadora por dos causas principales. En primer lugar, la mercantilizacio\#n- meritocratizacio\#n de la educacio\#n superior nos esta\# llevando a concebir a la educacio\#n como un bien consumible que permita, fa\#cil y ra\#pidamente, legitimar "conocimientos" como investigadores educativos, aunque la realidad es otra completamente, esto sucede por la complacencia entre el sector educativo privado que oferta este tipo de posgrados, y la Secretari\#a de Educacio\#n Pu\#blica que los avala a trave\#s del RVOE. Es lamentable vivir en una sociedad donde se privilegie los ti\#tulos nobiliarios por encima de los conocimientos y habilidades desarrolladas para mejorar la educacio\#n a nivel regional. Por parte del sector educativo pu\#blico, las universidades de Chiapas cada vez cuentan con menos apoyos

para formar profesionales de la investigacio\#n educativa, en especi\#fico, como causa de los asiduos recortes presupuestales que han tenido el Consejo Nacional de Ciencia y Tecnologi\#a (CONACYT) para con las becas de estudiantes de posgrados en el PNPC. Es difi\#cil calificar con guarismos la situacio\#n chiapaneca; sin embargo, mi querido Fabia\#n, te exhorto a que con el breve panorama que planteo de la educacio\#n superior en Chiapas, respondas a dicha interrogante.

F. R. J. Una de tus investigaciones recientes habla sobre la alarmante proliferacio\#n de iniciativas que ven la educacio\#n como un negocio; en este sentido, ¿que\# has descubierto al analizar de cerca el feno\#meno de la "credencializacio\#n" educativa en Chiapas?

L. A. A. G. Es lamentable aseverar que la credencializacio\#n de la educacio\#n es prioridad en los intereses y necesidades formativas de la sociedad chiapaneca, sea por las "mejoras" laborales que e\#sta trae consigo, o bien como medio para conseguir un empleo ma\#s remunerado, esto a pesar que en los u\#ltimos an\#os la OCDE ha concluido que en Me\#xico, a mayor grado de estudios menores posibilidades de trabajo existen. Sin embargo, la urdimbre econo\#mica sigue manteniendo a la educacio\#n como uno de los principales hilos en los que se sustenta; tal situacio\#n ha trai\# do consigo, sea como dan\#o colateral o bu\#squeda intencionada, que la educacio\# $\mathrm{n}$ - en te\#rminos de titulacio\#n acade\#mica- se le conciba como un bien de consumo, "necesario" para la mejora de la calidad y estilo de vida de los individuos. Altamente probable es que de esta premisa, grandes inversionistas nacionales y extranjeros han visualizado y apostado a la creacio\#n de instituciones educativas, con mayor e\#nfasis en las Instituciones de Educacio\#n Superior (IES), como empresas con alta plusvali\#a cuya derrama puede superar otros negocios mercantiles cuya intencio\#n sea la obtencio\#n ingente de ganancias: en Chiapas es muy evidente esta situacio\#n, en primer lugar por la proliferacio\#n desmedida de la creacio\#n de IES particulares y, por otro lado, por la gran demanda 
de dicho servicio, donde los principales consumidores son los docentes de educacio\#n ba\#sica del estado.

Por ello, es necesario cuestionarse el verdadero sentido que el te\#rmino calidad de la educacio\#n esta\# significando dentro de las poli\#ticas pu\#blicas educativas nacionales, ya que muy lejos queda de lograrse el objetivo de "formar capital humano de calidad", cuando en la realidad se esta\# mercantilizando la educacio\#n a pasos agigantados, mercantilizacio\#n educativa en tanto actividad generadora de riquezas para duen\#os y socios de las IES privadas del estado de Chiapas, y como medio para deslindar responsabilidades y obligaciones concatenadas al oficio del Estado Nacio\#n como lo es Me\#xico. En este sentido, bien cabe preguntarse, ¿cua\#l es el propo\#sito real de la educacio\#n en el pai\#s? ¿La formacio\#n humanista que se pregona en el Nuevo Modelo Educativo 2017 o, por el contrario, la sen\#era creacio\#n de necesidades educativas consumibles?

F. R. J. La vinculacio\#n con investigadores de renombre, propuesto por la revista electro\#nica Sinergias Educativas, se concreto\# exitosamente con la realizacio\#n de ambos cursos. ¿Que\# otras li\#neas de trabajo tienen proyectadas, adema\#s de la publicacio\#n del nu\#mero cero de la revista?

L. A. A. G. Como bien lo mencionas estimado Fabia\#n, la revista electro\#nica Sinergias Educativas tiene previsto publicar el nu\#mero cero para finales de septiembre del presente an\#o. Este es un evento de gran importancia y trascendencia para nosotros, tanto en el aspecto intelectual como en el emotivo, ya que con esta publicacio\#n iniciamos formalmente un proyecto que he disen\#ado y construido durante seis meses y que hasta el momento

Por otro lado, Sinergias Educativas sera\# organizadora y co-convocante en el I Congreso Internacional de Educacio\#n “Tendencias, reformas y modelos educativos a debate desde la investigacio\#n educativa”, que se llevara\# a cabo del 14 al 16 de junio de 2018 en la ciudad de Puebla de Zaragoza, Me\#xico, evento realizado gracias al intere\#s y apoyo de la Universidad de Oriente por aperturar estos espacios para la presentacio\#n $\mathrm{y}$ discusio\#n de investigaciones que responden a las problema\#ticas educativas a nivel internacional.

Asimismo, hay otras actividades de gran relevancia para Sinergias Educativas; empero, por estar en proceso de disen\#o y maduracio\#n reflexiva, prefiero mantenerlas al margen hasta que cuente con la certeza para darlas a conocer. Esta revista fue disen\#ada con la intencio\#n, a largo plazo, de posicionarse como referente nacional e internacional en el campo de la investigacio\#n educativa, ten por seguro mi querido amigo que lo lograremos gracias al apoyo de todo el equipo editorial que respalda este proyecto. 